\title{
From milk to malignancy: the role of mammary stem cells in development, pregnancy and breast cancer
}

\author{
Benjamin Tiede ${ }^{1}$, Yibin Kang ${ }^{1,2}$ \\ ${ }^{I}$ Department of Molecular Biology, Princeton University, Washington Road, Princeton, NJ 08544, USA; ${ }^{2}$ Breast Cancer Program, \\ Cancer Institute of New Jersey, New Brunswick, NJ 08903, USA
}

\begin{abstract}
Adult stem cells of the mammary gland (MaSCs) are a highly dynamic population of cells that are responsible for the generation of the gland during puberty and its expansion during pregnancy. In recent years significant advances have been made in understanding how these cells are regulated during these developmentally important processes both in humans and in mice. Understanding how MaSCs are regulated is becoming a particularly important area of research, given that they may be particularly susceptible targets for transformation in breast cancer. Here, we summarize the identification of MaSCs, how they are regulated and the evidence for their serving as the origins of breast cancer. In particular, we focus on how changes in MaSC populations may explain both the increased risk of developing aggressive ER/PR(-) breast cancer shortly after pregnancy and the long-term decreased risk of developing ER/ PR(+) tumors.
\end{abstract}

Keywords: mammary stem cells; MaSC; pregnancy; breast cancer

Cell Research (2011) 21: 245-257. doi:10.1038/cr.2011.11; published online 18 January 2011

\section{Biology of the mammary gland}

The mammary gland is composed of epithelial, adipose and other stromal cells, which work in concert for the primary goal of producing milk during nursing. In the female mouse, five rudimentary pairs of mammary epithelial placodes begin to form from the ectoderm at E10.5 and grow until E18, at which point growth is relatively restricted until puberty [1]. This makes the mammary gland relatively unique among most tissues and organs in that the majority of its patterning occurs in adulthood. Once gland expansion resumes during puberty, the epithelium forms into a branching, bilayered ductal structure, consisting of an outer myoepithelial layer of cells, which contract to help excrete milk and an inner luminal cell layer. This inner layer is subdivided into ductal luminal cells, which line the inside of the ducts, and alveolar luminal cells, which secrete milk during lactation (Figure 1A). In the mouse, mammary gland

Correspondence: Yibin Kang

Tel: +1 609258 8834; Fax: +1 6092582340

E-mail: ykang@princeton.edu growth during puberty is led by the invasion of club-like structures at the end of the ducts known as terminal end buds (TEBs), which invade into the empty adipose tissue, dubbed the mammary fat pad. TEBs consist of an outer layer of "cap" cells, which eventually form myoepithelial cells, and an inner layer of "body" cells, which become the luminal cell compartment (Figure 1B). The TEBs lead the growth of the gland until they reach the end of the fat pad, at which point they disappear [2]. The rest of the space in post-pubertal mammary gland is taken up by adipose tissue, along with a mixture of blood vessels, immune cells and fibroblasts [3].

After the extensive ductal elongation during puberty, the mammary gland undergoes minor growth and involution during the stages of the estrus cycle until pregnancy, at which point the gland is massively remodeled. During pregnancy, branches extend off the side of mammary ducts and proliferate to form lobuloalveolar "buds" or "bunches" which secrete milk. During late pregnancy and into lactation, the mammary epithelium fills the majority of the mammary fat pad. Alveolar cells secrete milk into the lumens, which is forced through the ducts by the contractile force of myoepithelial cells. After pups are weaned, the mammary epithelial cells undergo wellchoreographed apoptosis, resulting in the involution of 

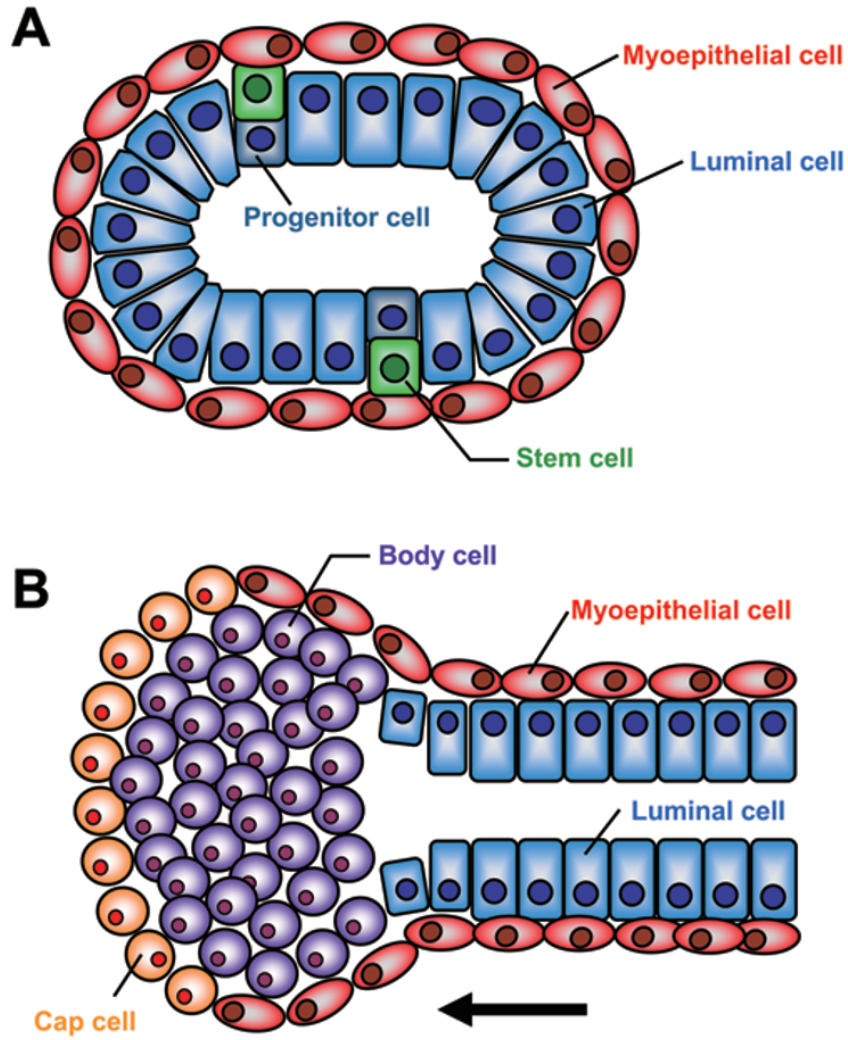

Figure 1 Cellular structures of the mammary gland. (A) The mature mammary duct features an outer layer of myoepithelial cells (red) surrounding an inner layer of luminal epithelial cells (blue). It is thought that mammary stem cells (green) reside in a basal position between these two populations and give rise to progenitor cells (teal) and both lineages of fully differentiated cells. (B) The developing mouse mammary gland invades through the empty fat pad led by the terminal end bud (TEB). Stem-like cap cells (orange) lead invasion in the direction of the black arrow and eventually give rise to myoepithelial cells. Many of the inner body cells (purple) undergo apoptosis as the gland grows, with some of the progeny of inner body cells forming the luminal cells that line the ducts of the glands.

the gland to the point where the parous gland returns to a structural state similar to the virgin gland. Upon the induction of further pregnancies, these coordinated growth and involution stages repeat in a similar fashion.

The gland architecture and remodeling that occurs in the human is highly similar to what is observed in the mouse mammary gland. However, there are some differences with respect to the gland structure. In humans the main lobular structure observed is known as the terminal ductal lobular unit (TDLU), which exists in several morphologically different forms throughout development and pregnancy. In the virgin gland, the relatively undifferen- tiated TDLUs are termed Lob1-type, which have been described as equivalent to TEBs [4]. As the Lob1-type TDLUs begin to develop and differentiate, they form Lob2-type TDLUs, which have more ductal structures per lobule compared to Lob1 structures. During pregnancy, the formation of even more ductules results in the conversion of Lob2 into Lob3, which eventually form secretory acinar structures (Lob4). After pregnancy, these TDLUs regress back in number, although in the resting parous gland the majority of TDLUs remain as Lob2 type, whereas in virgins Lob1 are the most predominant type [4-6]. In mature nulliparous women, Lob1 structures remain the most prevalent, with a moderate population of Lob2 structures but no Lob3 or Lob4 structures. After menopause, all women show a predominance for Lob1 structures, regardless of whether they have had children [4-6].

Because of the well-choreographed cycles of growth, remodeling and involution, researchers suspected for many years about the existence of adult stem cells within the mammary gland. These cells would theoretically be able to differentiate into the multiple cells of both the developing and pregnant gland, and self-renew despite the massive apoptosis post weaning to drive the growth of subsequent pregnancies. More dubiously, properties of mammary stem cells (MaSCs) could render them as vulnerable targets of tumorigenesis. The identity and characteristics of both the human and mouse MaSCs have been characterized in recent years, while their potential role during breast cancer formation is beginning to be elucidated.

\section{Identification of MaSCs}

The two hallmark properties of any stem cell population are the ability to differentiate into multiple cell lineages and the ability to self-renew to produce more stem cells [7]. While most adult cells are unable to divide, existing in a stage of terminal differentiation, tissue-specific adult stem cells retain the ability to divide and produce the multiple cell types within the organ from which they are derived. In the mouse mammary gland, the first clues for the existence of an adult stem cell of the mammary gland came from the work of DeOme et al. in the 1950s when it was shown that small pieces of mammary epithelium, when transplanted into recipient fat pads cleared of their endogenous epithelium, could expand and differentiate into a fully functional reconstituted gland [8]. Cells from nearly any location within the mammary gland, or during any developmental stage, could repopulate a mammary gland [9]. Subsequent experiments in both humans and mice demonstrated that this reconstitution 
ability was due to the activity of a single cell. Tsai et al. suggested clonal expansion was responsible for human mammary gland growth based on X-chromosome inactivation patterns [10], while Kordon and Smith demonstrated through retroviral tagging that mouse mammary glands were the progeny of a single cell [11]. Based on this evidence, a number of experimental approaches were undertaken to identify and purify MaSCs based on their biological or morphological properties.

One strategy to isolate MaSCs relies on a feature believed to be (although not universally accepted as) a key mechanism of DNA replication during stem cell division. As certain adult stem cells divide, they preferentially retain one of their DNA strands throughout multiple divisions in order to protect against the formation of deleterious mutations that occur during DNA replication $[12,13]$. By performing pulse-chase experiments with DNA labels, Smith et al. showed there was a population of cells within the mammary gland which retained their DNA label through asymmetric segregation of DNA strands. These cells were still actively dividing and featured stem cell characteristics [14]. Roughly $30-40 \%$ of the cells that retained their DNA label also expressed receptors for the reproductive hormones estrogen and progesterone [15]. As an early alternative approach to label retention, the heterogeneity of morphological characteristics of mammary epithelial cells was exploited to try to enrich for cells with stem cell characteristics. Pale cells with low cellular complexity (i.e. few cytoplasmic organelles) were shown to express the properties of MaSCs in differentiating conditions [9].

A major limitation of both the morphological and the label retention methods is that they do not lend themselves to the easy isolation of large numbers of relatively pure MaSC populations for use in in vitro or in vivo assays. As such, these methods did not definitively show that a single label-retaining cell or pale cell could reconstitute a fully functional gland in vivo, which is the gold standard for stem cell assays. A better approach to isolate putative MaSCs involved the simple isolation of different cell populations based on the expression of surface marker proteins from dissociated mammary gland preparations using fluorescence-activated cell sorting (FACS). An initial marker that showed some promise was stem cell antigen-1 or Sca1. Sca1 ${ }^{+}$cells were shown to be a subpopulation of the label-retaining epithelial cells, and when isolated they showed a degree of in vivo reconstitution ability. However, subsequent studies would identify markers which could enrich for MaSCs to a much higher degree of purity, and MaSCs identified by other methods have been shown to be Sca $1^{\text {low/- }}[7,16]$.

In 2006, mouse MaSCs were identified based on the expression of CD24 (heat-stable antigen) and high expression of either CD29 ( $\beta 1$-integrin) or CD49f ( $\alpha 6$ integrin) $[7,17]$. A single $\operatorname{Lin}^{-} \mathrm{CD} 24^{+} \mathrm{CD} 29^{\text {hi }} / \mathrm{CD} 49 \mathrm{f}^{\mathrm{hi}}$ cell was able to reconstitute an entire mammary gland in vivo. The CD29 protein is not just a surrogate marker for MaSCs but is actually functionally important, as CD29 ablation in the basal compartment reduced MaSC activity [18]. Multi-lineage differentiation of progenitors into luminal and myoepithelial cells was confirmed via histological analysis and a variety of in vitro differentiation assays. The second hallmark of stem cells, self-renewal, was confirmed via the observation of clonal gland outgrowth during serial gland reconstitution experiments. With respect to previous markers of stemness, Sca1 did not further enrich for the MaSCs, but the newly isolated MaSCs did seem to retain their DNA label [7]. Based on expression profiling and histological staining, the remaining non-stem cell fraction of the $\operatorname{Lin}^{-} \mathrm{CD} 24^{+} \mathrm{CD} 29^{\text {hi }}$ populations represents basal/myoepithelial cells. Downstream progenitor and mature luminal cells are primarily observed in the $\mathrm{CD} 24^{+} \mathrm{CD} 29^{\text {lo }}$ fraction. A specific luminal progenitor subpopulation of the $\mathrm{Lin}^{-} \mathrm{CD} 24^{+} \mathrm{CD} 29^{\mathrm{lo}}$ population has been identified based on strong expression of CD61 [19]. While the lineage of cells that differentiate to form the mammary gland has not been as well characterized as systems such as the colon, a more detailed description of the hierarchy of cells within the mammary gland can be found in the recent review by Visvader and Smith [20].

MaSCs are important for the two main growth phases of the mammary gland: the ductal elongation during pubertal expansion and the lobuloalveolar expansion during pregnancy. However, it is unknown whether the same population of cells with a high degree of plasticity can perform either of these functions depending on the local hormonal and growth cues, or if MaSCs begin to differentiate early and are programmed to perform only one of the two functions. There is evidence in other adult stem cell systems for the existence of two functionally distinct stem cell populations within one tissue [21]. Several lines of evidence support the assertion that this may be the case in the mouse mammary gland as well. Based on label retention studies, it was discovered that putative MaSCs existed in both basal and luminal locations [15]. With the subsequent identification of better surface marker profiles to efficiently purify MaSCs, stronger evidence emerged in parallel for the existence of distinct MaSC populations. In the MaSC fraction based on CD24 and CD49f staining, many cells expressed the basal maker K14. However, other cells expressed the luminal marker K18. It did not seem though that cells expressed both of these markers, suggesting that these cells might reside 
in distinct locations [17]. A luciferase-based transgenic mouse model for MaSC activity did reveal luciferaseexpressing cells in both basal and myoepithelial locations [22]. Notably, when the MaSC marker CD29 is deleted from the basal compartment of the mammary gland, the mammary epithelial cells can no longer reconstitute a new mammary gland, but they can form alveoli late in pregnancy [18], suggesting a distinct MaSC population. Similar results were also obtained when the Wnt receptor LPR5 was deleted [23]. A recent study showed that using a GFP reporter driven by the s-SHIP promoter, GFP ${ }^{+}$ replicating "active" MaSCs can be identified in cap cells in puberty and basal alveolar bud cells in pregnancy, but not in adult virgin animals, or in mammary tissues during lactation or involution stages [16]. Future characterizations will help to better understand whether or not distinct MaSC populations exist, and how they are controlled by their local micro-environmental cues.

With respect to the human mammary gland, identification of authentic MaSCs is a greater challenge because of the difficulty in obtaining normal tissue samples and the lack of an ideal in vivo reconstitution system. Nevertheless, various attempts have been made to characterize human MaSCs both in vitro and in vivo. By following similar methods of growing primary neural cells in nonadherent conditions which resulted in the formation of neural stem cell-enriched "neurospheres", mammary stem/progenitor cells could be enriched by forming "mammospheres" [24]. This method was further refined (for both human and mouse cells) by staining the mammary epithelial cells with the lipophilic dye PKH26 and selecting for cells that were slow dividing and retained this label during mammosphere growth. These cells were shown to have MaSC function in humanized mouse mammary glands in vivo [25]. An alternative isolation method was shown later by sorting cells based on the surface maker profile of $\mathrm{Lin}^{-} \mathrm{CD} 49^{+} \mathrm{EpCam}^{-/ 10}$ or $\mathrm{CD} 10^{+}$ and suspending these cells with irradiated human fibroblasts in a collagen gel and then implanting them under the kidney capsule of estrogen/progesterone-treated mice [26]. It was subsequently shown that this same population of cells could differentiate into mammary gland structures in mouse mammary glands when transplanted with supporting fibroblasts [27]. Expression profiling of human and mouse MaSCs-enriched populations has shown a significant degree of conservation in gene expression across species [28], providing validity to using the more readily accessible mouse model. Based on these enrichment methods for both human and mouse MaSCs, subsequent experiments have begun to elucidate the mechanisms by which MaSCs are controlled through various signaling pathways.

\section{Signaling pathways important for maintaining MaSCs}

A number of pathways that have been shown to play important roles in other adult stem cell systems also function in regulating MaSCs. For instance, in the Wnt pathway the receptor LRP5 can enrich for MaSCs on its own (although to a lesser degree than CD24 and CD29/ CD49f) and is functionally required for maintaining stem cell populations [23]. Overexpression of Wnt1 using the mammary-specific MMTV promoter resulted in a 6.4fold increase in the number of MaSCs [7]. Furthermore, Wnt ligands can be used to maintain MaSCs in culture, and when the Wnt pathway is stimulated in MaSCs they can outcompete untreated MaSCs in reconstitution assays [29]. Additionally, the Notch pathway has also been implicated in regulating MaSC fates. Multiple reports have shown that Notch pathway ligands are expressed in the MaSC compartment, while the Notch receptors are expressed in the downstream progenitor/luminal compartment $[25,30]$. Ablation of Notch signaling through a $C b f-1$ knockdown led to an expansion of MaSC activity, while forced constitutively active Notch signaling reduced MaSC activity [30]. As MaSCs divide in nonadherent growth conditions in vitro, the Notch antagonist Numb is asymmetrically localized into only one of the daughter cells. However, in MaSCs taken from a p53 mouse, Numb is ubiquitously localized in both daughter cells. Not surprisingly, there is an expansion of the MaSC population in $\mathrm{p} 53^{--}$mice [31], which seems to be the result of altered asymmetric DNA segregation and cell division rather than the anti-apoptotic activity of p53 [32]. Intriguingly though, inhibition of the Notch pathway in $\mathrm{p} 53^{--}$mammary epithelial cells reduced the MaSC activity [32], suggesting that further research is needed to better understand the role of Notch in MaSC growth and differentiation. Finally, the p53-related protein p63, a transcription factor known to be important for stem cell function and epithelial stasis in other systems [33, 34], is also important for MaSCs.

p63 has previously been shown to be important for maintaining the replicative potential of basally located stem cells in the epidermis, rather than functioning in lineage commitment or differentiation programs [34, 35]. p63 is expressed in primarily two different isoforms, $\Delta \mathrm{N}-\mathrm{p} 63$ and TA-p63. The $\Delta \mathrm{N}-\mathrm{p} 63$ isoform is expressed in MaSCs of the human [27] and mouse [36, 37], where its expression can be induced by Wnt signaling [23] and promotes the expression of self-renewal genes. The TAp63 isoform is expressed in luminal progenitor populations and promotes the expression of hedgehog pathway components necessary for progenitor cell function [37]. 
Recently, an interesting connection between p63 and Notch signaling has emerged. As mentioned, it had been shown that MaSCs are Notch signal-sending cells, expressing the ligands on their surface, while mammary progenitors are Notch signal-receiving cells [30]. This pattern of expression is opposite to that of the basally expressed p63. Through the use of a Notch reporter transgenic mouse it was shown that Notch and p63 were distinctly segregated from one another [38]. Intriguingly, these genes seem to be functionally antagonistic to one another. $\Delta \mathrm{N}-\mathrm{p} 63$ expression is necessary and sufficient for maintaining cells in the basal lineage, but when the activated intracellular intermediate of Notch1 (NICD) is overexpressed, it lowers the expression of $\Delta \mathrm{N}-\mathrm{p} 63$ in cells differentiating into the luminal lineage [38]. Furthermore, in Notch signaling-deficient RBP-J knockout mice, $\Delta \mathrm{N}$-p63 is aberrantly expressed in luminal cells [39], which may explain why MaSCs are known to be expanded in Notch-deficient mice [30].

\section{Mammary epithelial cell dynamics during develop- ment and pregnancy}

The ductal growth observed during puberty and the alveolar expansion that takes place during pregnancy are the two main periods of extensive mammary epithelial proliferation. Recently, evidence has suggested that these changes are driven by the coordinated division and differentiation of mammary stem/progenitor cell populations. MaSCs localized in the cap region of the TEB are responsible for the growth during the ductal elongation phase in puberty $[7,17]$ (Figure 1B). New data have also supported the notion that MaSCs are important for the growth observed during pregnancy. Intriguingly, an apoptosis-resistant population of cells known as parityinduced mammary epithelial cells (PI-MECs), which arise during pregnancy after activation of the whey acidic protein (WAP) promoter, show stem cell characteristics $[40,41]$. PI-MECs are a heterogeneous population, but generally express the MaSC markers CD24 and CD49f, and when transplanted can reconstitute mammary glands.

Further analysis based on both non-invasive in vivo tracking [22] or FACS analysis combined with gland reconstitution assays $[22,42]$ has shown a significant expansion both in total number and in percentage of MaSCs during pregnancy. The peak of this expansion seems to occur mid- to late-pregnancy at the end of alveolar proliferation and the onset of differentiation to begin milk production. Although s-SHIP promoter-driven GFP reporter marks active MaSCs in both the puberty and pregnancy stages [16], it is unknown whether pregnancyassociated MaSCs come from the same stem cell popula- tion important for pubertal growth, or if a distinct MaSC population exists or is responsible specifically for growth during pregnancy. While the start of milk production corresponds with the drop in MaSC numbers, sustained milk production continues to affect the rate of MaSC apoptosis, as when mothers do not nurse their young MaSC numbers drop quicker than those who do nurse their young [22]. Eventually, well after weaning of pups, a statistically significant reduction in the percentage of MaSCs has been observed in young but not old mice [22, $43,44]$. During pregnancy, MaSC expansion comes at a cost of self-renewal capability. This was demonstrated by Asselin-Labat et al. [42], who showed a defect in secondary gland reconstitution from donor cells taken from pregnant mice. Thus, it is not surprising that by tracking MaSCs in individual mice using an in vivo model, a smaller expansion of MaSCs has been observed in second pregnancies [22].

Downstream of MaSCs, the progenitor/luminal population also expands extensively and then involutes, but with an understandably delayed onset compared to the MaSCs [22]. By refining this population based on CD61 staining, it has been shown that the $\mathrm{CD} 61^{+}$progenitors do not rise in total number until later in pregnancy during lactation [19, 42], which suggests that the first increase in the combined $\mathrm{CD} 24^{+} \mathrm{CD} 29^{\text {lo }}$ population observed before pups are born [22] is likely to be $\mathrm{CD}^{-} 1^{-}$cells. Since $\mathrm{CD}^{+} 1^{+}$progenitors do not express hormone receptors but $\mathrm{CD}^{-} 1^{-}$cells do [19], the initial rise of $\mathrm{CD} 61^{-}$cells is likely a direct effect of the hormone signaling, but the second wave of $\mathrm{CD} 61^{+}$proliferation is likely the result of division of MaSCs into progenitors.

Given that mammary stem/progenitor cell dynamics change extensively during puberty and pregnancy, it is not surprising that the reproductive hormones estrogen, progesterone and prolactin play important roles in this regulation. In general, estrogen signaling is important for ductal elongation during puberty [45] and also during the early stages of pregnancy [40]; progesterone is also important for the initial side branching observed early in pregnancy [46] and prolactin is important for differentiation late in pregnancy [47]. In each of these cases, indirect paracrine signaling was shown to be important for the expansion of the mammary gland, which is not surprising since MaSCs and progenitors have been shown to not express receptors for these hormones $[19,36]$ and hormone receptor-positive cells generally do not readily proliferate [48]. A number of regulators and downstream targets of these hormones have been identified that control the growth and differentiation of MaSCs and progenitors. For instance, the prolactin target STAT5A is necessary and sufficient for the formation of $\mathrm{CD} 61^{+}$ 
luminal progenitors in virgin mice $[49,50]$ while targets ELF5 and GATA-3 are important during differentiation of $\mathrm{CD} 1^{+}$luminal progenitors during pregnancy $[19,51$, 52]. With respect to MaSCs, the progesterone receptor regulator $\mathrm{C} / \mathrm{EBP} \beta$ is needed to maintain the MaSC pool in virgin mice, and is also important during pregnancy [53, 54].

Another pathway that seems to be particularly important for MaSC expansion during pregnancy is the RANK/NF- $\kappa$ B pathway. It has been known that the pregnancy-associated hormones progesterone, prolactin and PTHrP increase the expression of the RANK ligand (RANKL) [55] while knockout of RANK, RANKL or inhibition of the downstream kinase IKK results in a defect in lobuloalveolar expansion and milk secretion during pregnancy $[55,56]$. This defect is largely due to impaired activity of the cell cycle-promoting factor Cyclin D1 [56]. Recent evidence has begun to suggest this effect is specific to MaSCs. Through expression profiling, it was shown that RANK expression is elevated in MaSCs during pregnancy or in response to hormone treatment, while RANKL is expressed in luminal cells [42]. This leads to the activation of the stem cell factor ID2 in MaSCs. When pregnancy hormones were removed via ovariectomy, a loss of RANK signaling was observed, as well as a significant loss in the number of functional MaSCs [42]. Notably, RANK inhibition directly limited the colony-forming ability of sorted MaSC populations, suggesting a functional role [42].

Given this evidence for the role of RANK/NF- $\kappa B$ signaling in promoting alveolar cell proliferation through activation of MaSCs, it is not surprising that this pathway is often associated with breast cancer formation [57]. Furthermore, this could help explain the propensity for breast cancer metastasis to bone, since this pathway constitutes an important part of the "vicious cycle" of osteolytic bone metastasis [58]. Unfortunately, this pathway is not alone among MaSC-regulatory factors that are also important drivers of tumorigenesis. As such, mounting evidence implicates a role for MaSC growth during breast cancer formation.

\section{MaSCs and breast cancer}

Because of their relatively long life span and ability to undergo self-renewing divisions, adult stem cells have been suggested as ideal candidates for the initial transforming events that drive cancer formation. While this has been demonstrated for leukemia [59], evidence in solid tumors has not been as clear cut. It is important to remember that breast cancer is a heterogeneous set of diseases, each with their own etiology, course of progression and outcome. Nevertheless, it is possible that MaSCs could serve as the cell of origin for distinct classes of breast cancer. Based on clustering analysis of gene expression data from a cohort of human breast cancers, five major tumor types were identified $[60,61]$. The basal type of tumors is frequently (but not always) "triple negative" for the expression of ER, PR and ErbB2/Neu. Clinically this subtype of tumors is of great interest because it is associated with a poor patient prognosis [60, 61]. MaSCs also lack the expression of these receptors [36], suggesting that they may be the tumor-initiating cell. Additionally, a number of previously identified regulators expressed by MaSCs such as Notch ligands, p63 and components of the Wnt pathway are known to be involved in basal-type tumors and are associated with poor outcomes [62-64]. Furthermore, markers of the epithelial-mesenchymal transition (EMT) are preferentially expressed in the basal and claudin-low subtypes of breast cancer [65-67]. Notably, multiple facets of the EMT process are thought to play an important role in expansion and invasion of the MaSC-rich TEBs during development [68], and it was also shown that forced EMT produces mammary cells with stem cell and tumorigenic characteristics [69]. More substantial investigation has further implicated that transformation of mammary stem or progenitor cells might drive tumorigenesis for distinct tumor types and ultimately effect patient prognosis. Much of this evidence fits into the paradigm of tumorinitiating cancer stem cells (CSCs).

It is believed that tumors, much like mature tissues and organs, are comprised of a hierarchy of cells which contain differing degrees of replicative and differentiation capacity. CSCs are the subpopulation of a heterogeneous tumor, which when separated and transplanted can selfrenew and differentiate into tumors matching the initial degree of heterogeneity. The remaining tumor cells are devoid of this property. By determining the identity of the original cell population that was transformed to form the CSC population, researchers will be able to identify key steps early in the formation of various cancers, and potentially reveal novel therapeutic targets which would allow for analyzing the root cause of tumor formation.

Multiple features of stem/progenitor cells make them likely candidates as the origin of CSCs. Stem/progenitor cells generally are very long lived compared to committed cell populations, which would provide a greater window for them to accumulate the multiple genetic mutations necessary for transformation. Furthermore, the self-renewal capability that stem/progenitor cells possess predisposes them with the replicative potential needed for overt tumor formation. Although stem cells often have mechanisms to protect against DNA damage 
(such as asymmetric segregation of DNA strands), loss of these protective mechanisms is often a hallmark of tumor formation. Several lines of evidence suggest that CSCs exist in tumors of other tissues/organs and may be derived from stem/progenitor cells (reviewed in [70-72]); however, only evidence concerning breast CSCs will be discussed here.

In a landmark paper in 2003, Al Hajj et al. showed that a subpopulation of human tumor cells with the surface marker expression profile of $\mathrm{CD} 44^{+} \mathrm{CD} 24^{-/ 10}$ could form heterogeneous tumors in serial transplantation assays [73]. However, given the multiple mechanisms that can drive breast cancer initiation, there are likely to be multiple different CSC populations with potentially different cells of origin depending on the oncogenic event $[7,74]$. Mouse models provide a simple way to evaluate different transformation events. In $\mathrm{p} 53^{-/-}$mice, there are elevated numbers of MaSCs [31], and the MaSC marker profile $\mathrm{CD} 24^{+} \mathrm{CD} 29^{\text {hi }}$ can enrich for breast CSCs [75]. However, in the MMTV-Wnt1 and $\mathrm{p} 53^{+/-}$models, the mammary progenitor marker CD61 enriched for breast CSCs [74]. Intriguingly, the $\mathrm{CD} 61^{+}$cells in the MMTV-Wnt1 tumors showed some mammary repopulating ability normally reserved for stem cell populations, suggesting that a readoption or improper expression of stem cell characteristics could be an early event during tumorigenesis in this model. This could also explain the expansion of MaSCs observed in pre-neoplastic tissue in this strain [7]. Breast CSCs were also identified in MMTV-Wnt 1 mice by the expression of CD24 and Thy1 (Thy1 has been used as a stem cell marker in other tissues and organs). When profiled, these cells produced a signature that was similar to published MaSC gene expression signatures [76]. Other global gene expression profiling analyses suggested that the mouse MaSC signature correlated with not only the MMTV-Wnt1 tumors, but also $\mathrm{p} 53^{-/-}$tumors [28]. The $\mathrm{CD} 1^{+}$luminal progenitor population most closely resembled MMTV-Neu and MMTV-PyMT tumors, while committed luminal cells resembled MMTV-Myc tumors [28].

As for human breast tumors, a variety of evidence suggests that MaSCs or progenitors may serve as the targets for transformation. Because of the difficulty in performing transplantation experiments of pre-neoplastic tissue with human tissues, gene expression profiling has often been used as a surrogate to show common expression patterns between normal mammary epithelial cells and particular tumor types, suggesting a possible cell of origin. For instance, by globally profiling the miRNAs that are differentially expressed between human breast CSCs and non-CSCs, a set of miRNAs were identified that are also differentially regulated between normal mammary gland stem/progenitor cells and committed cells [77]. In particular, mir-200 was expressed in the non-stem cell populations which suppressed Bmil expression that is needed to promote self-renewal and block differentiation [77]. Alternatively, a normal human MaSC signature was shown to be up-regulated in either undifferentiated or basal-like tumors [25]. However, using a different MaSC isolation method, it was shown that MaSCs showed overlap in gene expression profiles with claudin-low/normal breast-like tumors, while the luminal progenitors correlated highly with the basal cancer and committed luminal cells looked most like the luminal subtype tumors [27]. In the same study, Lim et al. were able to perform transplantation experiments from pre-neoplastic tissue similar to the experiments performed with mouse models by taking tissues from breast cancer-susceptible Brcal mutation carriers. It was suggested that in Brcal carriers luminal progenitor cells served as the targets for tumorigenesis [27]. Both BRCA1 and BRCA2 have previously been implicated in the normal differentiation process of the mammary gland $[78,79]$. Interestingly, in the Lim et al. study, the Brcal mutation carriers had lower MaSC numbers but higher numbers of luminal progenitors in normal glands. However, the progenitors from Brcal mutation carriers showed higher colony-forming ability than non-carriers (and even showed higher colony-forming ability than MaSCs in the absence of the growth supplement B27), suggesting an altered mammary hierarchy resulting from either stem or progenitor cell dysfunction.

In addition to these experimental studies, a variety of long-term observational studies have revealed associations suggesting that MaSCs or progenitor cells play an important role during tumorigenesis. For instance, many years after the massive radiation exposure of the nuclear bombs dropped on Hiroshima and Nagasaki, the cohort of women who showed the highest incidence of subsequent breast cancer development were those entering puberty at the time of the bombs, when MaSC activity is expected to be elevated $[80,81]$. Additionally, breast cancer was the most frequent cancer in women who received chest irradiation during adolescence for treatment of Hodgkin's disease [82, 83]. Some of the strongest observational evidence, though connecting mammary stem/ progenitor cells with breast cancer, focuses on the role of pregnancy in affecting breast cancer susceptibilities.

\section{Pregnancy, breast cancer and MaSCs}

For many years, epidemiological studies have demonstrated that an early, full-term pregnancy at young age is the only feature known to lower the lifetime risk of breast cancer without respect to race or ethnicity [4, 
84-87]. Women who are younger than 24 years old at the time of their first full-term pregnancy are protected against developing breast cancer much later in life, while women over the age of 35 are ultimately at an increased risk [88, 89]. However, immediately following the first full-term birth, women are at an increased risk of developing breast cancer [90], which in younger women lasts around 10 years, but in older women lasts longer [91]. Pregnancy generally protects against the development of ER/PR $(+)$ tumors, while the tumors that form shortly after pregnancy usually do not express these hormone receptors $[84,92,93]$ and are generally more aggressive. Because pregnancy results in such a strong, universal long-term protective effect against developing breast cancer, understanding the mechanism behind this effect could provide ideal targets to mimic this natural protective mechanism. Fortunately, this phenomenon is also observed in a variety of rodent models of chemically induced mammary tumors [4, 94], opening avenues to pursue experimental channels to understand the mechanism.

In general, there are four somewhat overlapping explanations to account for the protective effect (reviewed in [84]), all of which involve the impact on MaSCs to varying degrees (and are not mutually exclusive). Pregnancy could alter the levels of circulating hormones within the mammary gland, alter the hormone responsiveness of the cells within the gland itself, promote a more differentiated, growth-refractory state of the gland as a whole, or alter the number of MaSCs which could serve as the targets for transformation. Here, we will focus primarily on the role of MaSCs as the targets for transformation and how this may lead to an increased risk of developing aggressive ER/PR(-) tumors shortly after pregnancy and a decreased risk of developing ER/PR $(+)$ tumors long after weaning (Figure 2).

Two recent reports have shown in mouse models that a significant expansion of MaSCs occurs during pregnancy, peaking before the time that pups are born $[22,42]$. A number of features of tumors that form shortly after pregnancy suggest that direct transformation and expansion of MaSCs may drive the formation of these tumors. As mentioned above, pregnancy-associated tumors are ER/PR(-), similar to MaSCs themselves. Furthermore, tumors forming shortly after pregnancy in humans show elevated Her2 levels while showing decreased expression of the cell cycle inhibitor p27 and decreased levels of the p27 inducer BRCA1 [95]. Numerous pieces of evidence suggest that these may be critical effectors in transforming MaSCs during pregnancy.

With respect to Her2/Neu, tumors from MMTV-Neu transgenic mice are ER( $(-)$ and are composed of a fairly homogeneous population of luminal cells. Intriguingly,
A

Non-pregnant gland Breast cancer risk: average
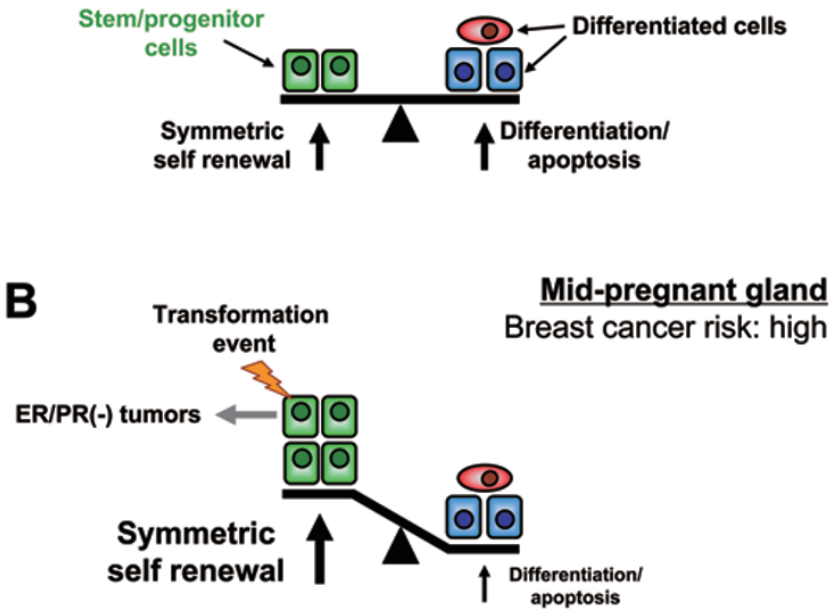

C

Post-pregnant Gland Breast cancer risk: low

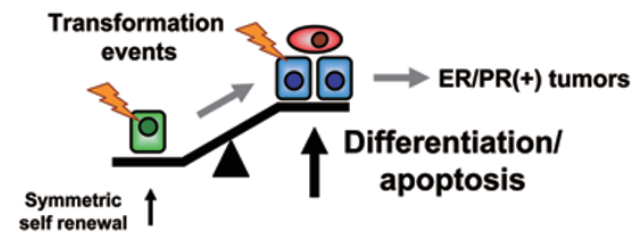

Figure 2 Potential roles of MaSCs during pregnancy and tumorigenesis. (A) In the normal virgin mammary gland, ER/PR(-) MaSCs (green) exist in a relative balance with $\mathrm{ER} / \mathrm{PR}(+)$ mature cells (blue, red). (B) During pregnancy, the number of MaSCs expands due to symmetric self-renewing divisions. This large pool of MaSCs may serve as the direct targets of transformation for ER/PR(-) tumors, leading to a short-term increased risk of breast cancer. (C) After weaning, through differentiation and involution, the number of MaSCs is lower than the resting gland and the remaining MaSCs have lower self-renewal ability. This lowers the overall risk for developing $\operatorname{ER/PR}(+)$ breast cancers, which may result either from transformation of MaSCs, which as they differentiate require additional oncogenic mutations to compensate for the reduced self-renewal ability before forming tumors, or from the direct transformation of $\mathrm{ER} / \mathrm{PR}(+)$ committed cells.

pregnancy-associated tumors which arise in the MMTV$\mathrm{Neu}$ strain are derived from the stem cell-containing PIMEC population [96]. Within the MMTV-Neu tumors, there are cells that could divide and produce cells which simultaneously expressed both luminal and myoepithelial cytokeratin markers, even though the tumors themselves were primarily luminal [97]. This suggested that 
the tumors retained some degree of stem/progenitor cell activity, which is understandable given that MMTV-Neu tumors show a loss of p53 which is linked to deregulated stem cell asymmetric division [31].

One critical mediator of Neu-driven pregnancyassociated breast cancer that affects MaSCs is Cyclin D1. Cyclin D1 knockout mice show defects in lobuloalveolar development and lactation during pregnancy and are refractory to tumor development when crossed with the MMTV-Neu strain [98-100]. The inactive Cyclin D1 K112E mutant shows a specific defect in PI-MEC cell self-renewal and differentiation during pregnancy [101], suggesting lower numbers of cells susceptible to tumor development. Not surprisingly, this strain also inhibits MMTV-Neu-driven tumor formation [101]. While mammary glands from these mice showed lower mammary reconstitution frequency, it appeared there was a specific defect in colony-forming progenitor cells, rather than MaSCs themselves [101]. Because the transgenic MMTV promoter for these experiments is pregnancy hormone responsive, the data observed with the MMTV$\mathrm{Neu}$ mice in relation to pregnancy should be interpreted cautiously until further confirmation is shown through more advanced knockout, conditional overexpression or lentiviral overexpression experiments.

In addition to Her2/Neu, another factor important for MaSCs that may help explain the increased risk of developing aggressive tumors shortly after pregnancy is BRCA1. BRCA1 tumors are typically ER/PR negative basal-type and tumor-initiating CSCs from BRCA1 mutation carriers can be isolated using markers of normal MaSCs [102]. BRCA1 is expressed in MaSC-enriched TEBs of the developing mouse mammary gland and its expression is elevated during pregnancy by combined stimulation of estrogen and progesterone [78]. Functionally, BRCA1 knockdown led to an increased amount of secondary and tertiary mammosphere formation and led to improper differentiation in vivo [103]. Not surprisingly, BRCA1 levels are reduced by $33 \%$ and p 27 levels are reduced by $89 \%$ in pregnancy-associated breast cancers [95]. Taken together, these observations suggest that changes in p53, Her2/Neu and BRCA1 expression in the expanded MaSC compartment may contribute to the increased risk of developing tumors shortly after giving birth (Figure 2B). These and other factors may also play a role in changing MaSC levels to protect against developing breast cancer long after pregnancy.

As mentioned above, many years after giving birth at young age, women develop ER/PR $(+)$ breast cancers at a decreased rate compared to women who have not undergone a full-term pregnancy. Multiple studies have confirmed that in young mice, pregnancy ultimately decreas- es the number of MaSCs in the mammary gland $[22,44]$. Again, if MaSCs are the targets for transformation, this could help explain the lower breast cancer rates in women having undergone a full-term pregnancy. Notably, this effect dissipates with increased age at the time of first pregnancy $[88,104]$, and in the mouse model pregnancy in older mice did not result in decreased MaSC numbers [43]. However, unlike the tumors that form shortly after pregnancy, the protective effect long after pregnancy is against $\mathrm{ER} / \mathrm{PR}(+)$ tumors, suggesting that in such cases mutations that occur in MaSCs may not directly lead to transformation, but could lead to tumor formation upon further oncogenic challenges in downstream progenitors (Figure 2C). This may partly be explained by the observation of altered self-renewal in MaSC populations after one full-term pregnancy and smaller expansion in successive pregnancies $[22,42]$. This suggests that the cells that remain after involution of the gland are not as growthcompetent and thus it may take more oncogenic insults to form tumors. A similar change in the characteristics of MaSCs after pregnancy has been proposed in human mammary glands, where pregnancy is known to result in the progression of Lob1 TDLUs into more differentiated Lob2, Lob3 and Lob4 structures. Russo et al. suggested that pregnancy results in a conversion of "Stem Cells 1" found in the undifferentiated Lob1 structures into more differentiated "Stem Cells 2" (roughly equivalent to mouse PI-MECs) found in more differentiated structures [4] which are resistant to tumor formation. When tumors do arise in the more differentiated TDLUs, they are less malignant.

If lowering the number of MaSCs is crucial for the pregnancy-associated breast cancer protection, then deregulation of the apoptotic machinery in MaSCs during involution or promotion of stem cell growth should correlate with a loss of the protective effect. (It is already known that improper apoptosis after hormone level decrease during the estrus cycle promotes tumorigenesis in progenitor cells in a transgenic model [105].) Not surprisingly, p53 activity, which is important for the normal involution process after pregnancy, is required for paritydependent breast cancer protection, while loss of p53 leads to an increase in MaSC activity through increased symmetric cell divisions $[31,106]$.

\section{Perspectives}

With the discovery of new markers to better identify and track MaSCs, clear insights have been gained regarding the role of MaSCs during developmentally important processes such as puberty and pregnancy. However, our understanding of the role of MaSCs in tu- 
morigenesis remains opaque compared to other systems. In order to better elucidate this role, future studies will greatly benefit from attempts to further refine the makers used to isolate MaSC populations to better purify MaSC fractions (and possibly distinct fractions important for gland elongation during puberty versus gland expansion during pregnancy). Additionally, it will be important to be able to better track MaSC populations in vivo to directly test their susceptibility to transformation in particular forms of breast cancer. By doing so, researchers will be able to ascertain how regulating MaSC numbers during pregnancy ultimately impacts parity-associated breast cancer risk and also begin to determine which of the key signaling pathways important for MaSCs in development also play a role during tumorigenesis. These insights will be particularly important in coming years given that women are electing to have children later in life, which results in a decreased or mitigated protective effect. Therefore, research to understand the mechanisms behind the protective effect will hopefully lead to the ability to better predict breast cancer susceptibility in high-risk patient groups during pregnancy and potentially even strategies to induce or enhance this protective effect when needed.

\section{References}

1 Watson CJ, Khaled WT. Mammary development in the embryo and adult: a journey of morphogenesis and commitment. Development 2008; 135:995-1003.

2 Silberstein GB. Postnatal mammary gland morphogenesis. Microsc Res Tech 2001; 52:155-162.

3 Wiseman BS, Werb Z. Stromal effects on mammary gland development and breast cancer. Science 2002; 296:10461049 .

4 Russo J, Moral R, Balogh GA, Mailo D, Russo IH. The protective role of pregnancy in breast cancer. Breast Cancer Res 2005; 7:131-142.

5 Russo J, Rivera R, Russo IH. Influence of age and parity on the development of the human breast. Breast Cancer Res Treat 1992; 23:211-218.

6 Russo J, Russo I. Development of the human mammary gland. In: Neville M, Daniel C, eds. The Mammary Gland. New York: Plenum, 1987:67-93.

7 Shackleton M, Vaillant F, Simpson K, et al. Generation of a functional mammary gland from a single stem cell. Nature 2006; 439:84-88.

8 DeOme K, Faulkin L, Bern H, Blair P. Development of mammary tumors from hyperplastic alveolar nodules transplanted into gland-free mammary fat pads of female $\mathrm{C} 3 \mathrm{H}$ mice. Cancer Res 1959; 19:515-520.

9 Smith GH, Medina D. A morphologically distinct candidate for an epithelial stem cell in mouse mammary gland. $J$ Cell Sci 1988; 90 (Part 1): 173-183.

10 Tsai YC, Lu Y, Nichols PW, Zlotnikov G, Jones PA, Smith
HS. Contiguous patches of normal human mammary epithelium derived from a single stem cell: implications for breast carcinogenesis. Cancer Res 1996; 56:402-404.

11 Kordon EC, Smith GH. An entire functional mammary gland may comprise the progeny from a single cell. Development 1998; 125:1921-1930.

12 Cairns J. Somatic stem cells and the kinetics of mutagenesis and carcinogenesis. Proc Natl Acad Sci USA 2002; 99:1056710570.

13 Potten CS, Owen G, Booth D. Intestinal stem cells protect their genome by selective segregation of template DNA strands. J Cell Sci 2002; 115:2381-2388.

14 Smith GH. Label-retaining epithelial cells in mouse mammary gland divide asymmetrically and retain their template DNA strands. Development 2005; 132:681-687.

15 Booth BW, Smith GH. Estrogen receptor-alpha and progesterone receptor are expressed in label-retaining mammary epithelial cells that divide asymmetrically and retain their template DNA strands. Breast Cancer Res 2006; 8:R49.

16 Bai L, Rohrschneider L. s-SHIP promoter expression marks activated stem cells in developing mouse mammary tissue. Genes Dev 2010; 24:1882-1892.

17 Stingl J, Eirew P, Ricketson I, et al. Purification and unique properties of mammary epithelial stem cells. Nature 2006; 439:993-997.

18 Taddei I, Deugnier MA, Faraldo MM, et al. Beta1 integrin deletion from the basal compartment of the mammary epithelium affects stem cells. Nat Cell Biol 2008; 10:716-722.

19 Asselin-Labat ML, Sutherland KD, Barker H, et al. Gata-3 is an essential regulator of mammary-gland morphogenesis and luminal-cell differentiation. Nat Cell Biol 2007; 9:201-209.

20 Visvader JE, Smith GH. Murine mammary epithelial stem cells: discovery, function, and current status. Cold Spring Harb Perspect Biol 2010 Oct 6. doi:10.1101/cshperspect. a004879

21 Barker N, van Es JH, Kuipers J, et al. Identification of stem cells in small intestine and colon by marker gene Lgr5. $\mathrm{Na}$ ture 2007; 449:1003-1007.

22 Tiede BJ, Owens LA, Li F, DeCoste C, Kang Y. A novel mouse model for non-invasive single marker tracking of mammary stem cells in vivo reveals stem cell dynamics throughout pregnancy. PLoS One 2009; 4:e8035.

23 Badders NM, Goel S, Clark RJ, et al. The Wnt receptor, Lrp5, is expressed by mouse mammary stem cells and is required to maintain the basal lineage. PLoS One 2009; 4:e6594.

24 Dontu G, Abdallah W, Foley J, et al. In vitro propagation and transcriptional profiling of human mammary stem/progenitor cells. Genes Dev 2003; 17:1253-1270.

25 Pece S, Tosoni D, Confalonieri S, et al. Biological and molecular heterogeneity of breast cancers correlates with their cancer stem cell content. Cell 2010; 140:62-73.

26 Eirew P, Stingl J, Raouf A, et al. A method for quantifying normal human mammary epithelial stem cells with in vivo regenerative ability. Nat Med 2008; 14:1384-1389.

27 Lim E, Vaillant F, Wu D, et al. Aberrant luminal progenitors as the candidate target population for basal tumor development in BRCA1 mutation carriers. Nat Med 2009; 15:907913.

28 Lim E, Wu D, Pal B, et al. Transcriptome analyses of mouse 
and human mammary cell subpopulations reveals multiple conserved genes and pathways. Breast Cancer Res 2010; 12:R21.

29 Zeng YA, Nusse R. Wnt proteins are self-renewal factors for mammary stem cells and promote their long-term expansion in culture. Cell Stem Cell 2010; 6:568-577.

30 Bouras T, Pal B, Vaillant F, et al. Notch signaling regulates mammary stem cell function and luminal cell-fate commitment. Cell Stem Cell 2008; 3:429-441.

31 Cicalese A, Bonizzi G, Pasi CE, et al. The tumor suppressor p53 regulates polarity of self-renewing divisions in mammary stem cells. Cell 2009; 138:1083-1095.

32 Tao L, Roberts AL, Dunphy KA, Bigelow C, Yan H, Jerry DJ. Repression of mammary stem/progenitor cells by P53 is mediated by Notch and separable from apoptotic activity. Stem Cells 2010 Oct 22. doi:10.1002/stem.552

33 Kurata S, Okuyama T, Osada M, et al. p51/p63 Controls subunit alpha3 of the major epidermis integrin anchoring the stem cells to the niche. J Biol Chem 2004; 279:50069-50077.

34 Senoo M, Pinto F, Crum CP, McKeon F. p63 Is essential for the proliferative potential of stem cells in stratified epithelia. Cell 2007; 129:523-536.

35 Koster MI, Kim S, Mills AA, DeMayo FJ, Roop DR. p63 is the molecular switch for initiation of an epithelial stratification program. Genes Dev 2004; 18:126-131.

36 Asselin-Labat M, Shackleton M, Stingl J, et al. Steroid hormone receptor status of mouse mammary stem cells. $J$ Natl Cancer Inst 2006; 98:1011-1014.

37 Li N, Singh S, Cherukuri P, et al. Reciprocal intraepithelial interactions between TP63 and Hedgehog signaling regulate quiescence and activation of progenitor elaboration by mammary stem cells. Stem Cells 2008; 26:1253-1264.

38 Yalcin-Ozuysal O, Fiche M, Guitierrez M, Wagner KU, Raffoul W, Brisken C. Antagonistic roles of Notch and p63 in controlling mammary epithelial cell fates. Cell Death Differ 2010; 17:1600-1612.

39 Buono KD, Robinson GW, Martin C, et al. The canonical Notch/RBP-J signaling pathway controls the balance of cell lineages in mammary epithelium during pregnancy. Dev Biol 2006; 293:565-580.

40 Matulka LA, Triplett AA, Wagner KU. Parity-induced mammary epithelial cells are multipotent and express cell surface markers associated with stem cells. Dev Biol 2007; 303:2944.

41 Wagner KU, Boulanger CA, Henry MD, Sgagias M, Hennighausen L, Smith GH. An adjunct mammary epithelial cell population in parous females: its role in functional adaptation and tissue renewal. Development 2002; 129:1377-1386.

42 Asselin-Labat ML, Vaillant F, Sheridan JM, et al. Control of mammary stem cell function by steroid hormone signalling. Nature 2010; 465:798-802.

43 Britt KL, Kendrick H, Regan JL, et al. Pregnancy in the mature adult mouse does not alter the proportion of mammary epithelial stem/progenitor cells. Breast Cancer Res 2009; 11:R20.

44 Siwko SK, Dong J, Lewis MT, Liu H, Hilsenbeck SG, Li Y. Evidence that an early pregnancy causes a persistent decrease in the number of functional mammary epithelial stem cells - implications for pregnancy-induced protection against breast cancer. Stem Cells 2008; 26:3205-3209.

45 Mallepell S, Krust A, Chambon P, Brisken C. Paracrine signaling through the epithelial estrogen receptor alpha is required for proliferation and morphogenesis in the mammary gland. Proc Natl Acad Sci USA 2006; 103:2196-2201.

46 Brisken C, Park S, Vass T, Lydon JP, O’Malley BW, Weinberg RA. A paracrine role for the epithelial progesterone receptor in mammary gland development. Proc Natl Acad Sci USA 1998; 95:5076-5081.

47 Brisken C, Kaur S, Chavarria TE, et al. Prolactin controls mammary gland development via direct and indirect mechanisms. Dev Biol 1999; 210:96-106.

48 Beleut M, Rajaram RD, Caikovski M, et al. Two distinct mechanisms underlie progesterone-induced proliferation in the mammary gland. Proc Natl Acad Sci USA 2010; 107:2989-2994.

49 Hennighausen L, Robinson GW. Interpretation of cytokine signaling through the transcription factors STAT5A and STAT5B. Genes Dev 2008; 22:711-721.

50 Yamaji D, Na R, Feuermann Y, et al. Development of mammary luminal progenitor cells is controlled by the transcription factor STAT5A. Genes Dev 2009; 23:2382-2387.

51 Choi YS, Chakrabarti R, Escamilla-Hernandez R, Sinha S. Elf5 conditional knockout mice reveal its role as a master regulator in mammary alveolar development: failure of Stat5 activation and functional differentiation in the absence of Elf5. Dev Biol 2009; 329:227-241.

52 Oakes SR, Naylor MJ, Asselin-Labat ML, et al. The Ets transcription factor Elf5 specifies mammary alveolar cell fate. Genes Dev 2008; 22:581-586.

53 LaMarca HL, Visbal AP, Creighton CJ, et al. CCAAT/enhancer binding protein beta regulates stem cell activity and specifies luminal cell fate in the mammary gland. Stem Cells 2010; 28:535-544.

54 Seagroves TN, Lydon JP, Hovey RC, Vonderhaar BK, Rosen JM. C/EBPbeta (CCAAT/enhancer binding protein) controls cell fate determination during mammary gland development. Mol Endocrinol 2000; 14:359-368.

55 Fata JE, Kong YY, Li J, et al. The osteoclast differentiation factor osteoprotegerin-ligand is essential for mammary gland development. Cell 2000; 103:41-50.

56 Cao Y, Bonizzi G, Seagroves TN, et al. IKKalpha provides an essential link between RANK signaling and cyclin D1 expression during mammary gland development. Cell 2001; 107:763-775.

57 Cao Y, Luo JL, Karin M. IkappaB kinase alpha kinase activity is required for self-renewal of ErbB2/Her2-transformed mammary tumor-initiating cells. Proc Natl Acad Sci USA 2007; 104:15852-15857.

58 Lu X, Kang Y. Organotropism of breast cancer metastasis. $J$ Mammary Gland Biol Neoplasia 2007; 12:153-162.

59 Bonnet D, Dick JE. Human acute myeloid leukemia is organized as a hierarchy that originates from a primitive hematopoietic cell. Nat Med 1997; 3:730-737.

60 Perou CM, Sorlie T, Eisen MB, et al. Molecular portraits of human breast tumours. Nature 2000; 406:747-752.

61 Sorlie T, Perou C, Tibshirani R, et al. Gene expression patterns of breast carcinomas distinguish tumor subclasses with clinical implications. Proc Natl Acad Sci USA 2001; 
98:10869-10874.

62 Khramtsov AI, Khramtsova GF, Tretiakova M, Huo D, Olopade OI, Goss $\mathrm{KH}$. Wnt/\{beta $\}$-catenin pathway activation is enriched in basal-like breast cancers and predicts poor outcome. Am J Pathol 2010; 176:2911-2920.

63 Matos I, Dufloth R, Alvarenga M, Zeferino LC, Schmitt F. p63, cytokeratin 5, and P-cadherin: three molecular markers to distinguish basal phenotype in breast carcinomas. Virchows Arch 2005; 447:688-694.

64 Reedijk M, Pinnaduwage D, Dickson BC, et al. JAG1 expression is associated with a basal phenotype and recurrence in lymph node-negative breast cancer. Breast Cancer Res Treat 2008; 111:439-448.

65 Sarrio D, Rodriguez-Pinilla SM, Hardisson D, Cano A, Moreno-Bueno G, Palacios J. Epithelial-mesenchymal transition in breast cancer relates to the basal-like phenotype. Cancer Res 2008; 68:989-997.

66 Hennessy BT, Gonzalez-Angulo AM, Stemke-Hale K, et al. Characterization of a naturally occurring breast cancer subset enriched in epithelial-to-mesenchymal transition and stem cell characteristics. Cancer Res 2009; 69:4116-4124.

67 Taube JH, Herschkowitz JI, Komurov K, et al. Core epithelialto-mesenchymal transition interactome gene-expression signature is associated with claudin-low and metaplastic breast cancer subtypes. Proc Natl Acad Sci USA 2010; 107:1544915454.

68 Micalizzi DS, Farabaugh SM, Ford HL. Epithelial-mesenchymal transition in cancer: parallels between normal development and tumor progression. J Mammary Gland Biol Neoplasia 2010; 15:117-134.

69 Mani SA, Guo W, Liao MJ, et al. The epithelial-mesenchymal transition generates cells with properties of stem cells. Cell 2008; 133:704-715.

70 Clarke MF, Fuller M. Stem cells and cancer: two faces of eve. Cell 2006; 124:1111-1115.

71 Li F, Tiede B, Massague J, Kang Y. Beyond tumorigenesis: cancer stem cells in metastasis. Cell Res 2007; 17:3-14.

72 Wang JCY. Good cells gone bad: the cellular origins of cancer. Trends Mol Med 2009; 16:145-151.

73 Al-Hajj M, Wicha M, Benito-Hernandez A, Morrison S, Clarke MF. Prospective identification of tumorigenic breast cancer cells. Proc Natl Acad Sci USA 2003; 100:3983-3988.

74 Vaillant F, Asselin-Labat ML, Shackleton M, Forrest NC, Lindeman GJ, Visvader JE. The mammary progenitor marker CD61/beta3 integrin identifies cancer stem cells in mouse models of mammary tumorigenesis. Cancer Res 2008; 68:7711-7717.

75 Zhang M, Behbod F, Atkinson RL, et al. Identification of tumor-initiating cells in a p53-null mouse model of breast cancer. Cancer Res 2008; 68:4674-4682.

76 Cho RW, Wang X, Diehn M, et al. Isolation and molecular characterization of cancer stem cells in MMTV-Wnt-1 murine breast tumors. Stem Cells (Dayton, Ohio) 2008; 26:364371.

77 Shimono Y, Zabala M, Cho RW, et al. Downregulation of miRNA-200c links breast cancer stem cells with normal stem cells. Cell 2009; 138:592-603.

78 Marquis ST, Rajan JV, Wynshaw-Boris A, et al. The developmental pattern of Brcal expression implies a role in dif- ferentiation of the breast and other tissues. Nat Genet 1995; 11:17-26.

79 Rajan JV, Marquis ST, Gardner HP, Chodosh LA. Developmental expression of Brca2 colocalizes with Brca1 and is associated with proliferation and differentiation in multiple tissues. Dev Biol 1997; 184:385-401.

80 Tokunaga M, Land CE, Tokuoka S, Nishimori I, Soda M, Akiba S. Incidence of female breast cancer among atomic bomb survivors, 1950-1985. Radiat Res 1994; 138:209-223.

81 Land CE, Tokunaga M, Koyama K, et al. Incidence of female breast cancer among atomic bomb survivors, Hiroshima and Nagasaki, 1950-1990. Radiat Res 2003; 160:707-717.

82 Aisenberg AC, Finkelstein DM, Doppke KP, Koerner FC, Boivin JF, Willett CG. High risk of breast carcinoma after irradiation of young women with Hodgkin's disease. Cancer 1997; 79:1203-1210.

83 Swerdlow AJ, Barber JA, Horwich A, Cunningham D, Milan S, Omar RZ. Second malignancy in patients with Hodgkin's disease treated at the Royal Marsden Hospital. Br J Cancer 1997; 75:116-123.

84 Britt K, Ashworth A, Smalley M. Pregnancy and the risk of breast cancer. Endocr Relat Cancer 2007; 14:907-933.

85 Henderson BE, Powell D, Rosario I, et al. An epidemiologic study of breast cancer. J Natl Cancer Inst 1974; 53:609-614.

86 Kelsey J, Gammon M, John E. Reproductive factors and breast cancer. Epidemiol Rev 1993; 15:36-47.

87 D'Cruz CM, Moody SE, Master SR, et al. Persistent parityinduced changes in growth factors, TGF-beta3, and differentiation in the rodent mammary gland. Mol Endocrinol (Baltimore, MD) 2002; 16:2034-2051.

88 Lambe M, Hsieh C, Chan H, Ekbom A, Trichopoulos D, Adami H. Parity, age at first and last birth, and risk of breast cancer: a population-based study in Sweden. Breast Cancer Res Treat 1996; 38:305-311.

89 Trichopoulos D, Hsieh CC, MacMahon B, et al. Age at any birth and breast cancer risk. Int J Cancer 1983; 31:701-704.

90 Albrektsen G, Heuch I, Hansen S, Kvale G. Breast cancer risk by age at birth, time since birth and time intervals between births: exploring interaction effects. Br J Cancer 2005; 92:167-175.

91 Rosner B, Colditz GA, Willett WC. Reproductive risk factors in a prospective study of breast cancer: the Nurses' Health Study. Am J Epidemiol 1994; 139:819-835.

92 Hildreth NG, Kelsey JL, Eisenfeld AJ, LiVolsi VA, Holford TR, Fischer DB. Differences in breast cancer risk factors according to the estrogen receptor level of the tumor. $J$ Natl Cancer Inst 1983; 70:1027-1031.

93 Ruder AM, Lubin F, Wax Y, Geier A, Alfundary E, Chetrit A. Estrogen and progesterone receptors in breast cancer patients. Epidemiologic characteristics and survival differences. Cancer 1989; 64:196-202.

94 Medina D. Chemical carcinogenesis of rat and mouse mammary glands. Breast Dis 2007; 28:63-68.

95 Reed W, Sandstad B, Holm R, Nesland JM. The prognostic impact of hormone receptors and c-erbB-2 in pregnancyassociated breast cancer and their correlation with BRCA1 and cell cycle modulators. Int J Surg Pathol 2003; 11:65-74.

96 Henry MD, Triplett AA, Oh KB, Smith GH, Wagner KU. Parity-induced mammary epithelial cells facilitate tumori- 
genesis in MMTV-neu transgenic mice. Oncogene 2004; 23:6980-6985.

97 Liu JC, Deng T, Lehal RS, Kim J, Zacksenhaus E. Identification of tumorsphere- and tumor-initiating cells in HER2/Neuinduced mammary tumors. Cancer Res 2007; 67:8671-8681.

98 Fantl V, Stamp G, Andrews A, Rosewell I, Dickson C. Mice lacking cyclin D1 are small and show defects in eye and mammary gland development. Genes Dev 1995; 9:23642372.

99 Sicinski P, Donaher JL, Parker SB, et al. Cyclin D1 provides a link between development and oncogenesis in the retina and breast. Cell 1995; 82:621-630.

100 Yu Q, Geng Y, Sicinski P. Specific protection against breast cancers by cyclin D1 ablation. Nature 2001; 411:1017-1021.

101 Jeselsohn R, Brown NE, Arendt L, et al. Cyclin D1 kinase activity is required for the self-renewal of mammary stem and progenitor cells that are targets of MMTV-ErbB2 tumorigenesis. Cancer Cell 2010; 17:65-76.
102 Vassilopoulos A, Wang RH, Petrovas C, Ambrozak D, Koup $\mathrm{R}$, Deng CX. Identification and characterization of cancer initiating cells from BRCA1 related mammary tumors using markers for normal mammary stem cells. Int J Biol Sci 2008; 4:133-142.

103 Liu S, Ginestier C, Charafe-Jauffret E, et al. BRCA1 regulates human mammary stem/progenitor cell fate. Proc Natl Acad Sci USA 2008; 105:1680-1685.

104 Trichopoulos D, Hsieh C, MacMahon B, et al. Age at any birth and breast cancer risk. Int J Cancer 1983; 31:701-704.

$105 \mathrm{Li} \mathrm{Z,} \mathrm{Tognon} \mathrm{CE,} \mathrm{Godinho} \mathrm{FJ,} \mathrm{et} \mathrm{al.} \mathrm{ETV6-NTRK3} \mathrm{fusion}$ oncogene initiates breast cancer from committed mammary progenitors via activation of AP1 complex. Cancer Cell 2007; 12:542-558.

106 Gatza CE, Dumble M, Kittrell F, et al. Altered mammary gland development in the $\mathrm{p} 53+/ \mathrm{m}$ mouse, a model of accelerated aging. Dev Biol 2008; 313:130-141. 